\title{
Study of Molecular Interactions in Ternary Liquid Mixtures
}

\author{
Ch. Kavitha ${ }^{1, a *}$, A. Ratnakar ${ }^{1, b}$, K. Narendra ${ }^{2, c}$ \\ ${ }^{1}$ Department of Chemistry, V.R. Siddhartha Engineering College, Vijayawada, Andhra Pradesh 520 \\ 007, India. \\ ${ }^{2}$ Department of Physics, V.R. Siddhartha Engineering College, Vijayawada, Andhra Pradesh 520 \\ 007, India. \\ Email: a, ${ }^{*}$ chkavitha.chem@gmail.com, ${ }^{b}$ drabbineniratnakar@gmail.com, ${ }^{c}$ narenk75@gmail.com
}

Keywords: Density, quinoline, toluene, molecular interactions, ternary mixtures

\begin{abstract}
The ultrasonic velocities and densities of ternary mixtures of quinolone with toluene and methanol as common compound, including those of pure liquids, over the entire composition range were measured at temperatures $(303.15,308.15,313.15$ and 318.15$) \mathrm{K}$ and atmospheric pressure. From the experimental data, excess molar volume $V_{m}$, excess ultrasonic velocity $u^{\mathrm{E}}$ and excess acoustic impedance $\mathrm{Z}^{\mathrm{E}}$ were calculated. The variation these parameters with composition and temperature of the mixtures are discussed in terms of molecular interactions in these mixtures. All the calculated excess parameter values were found to be positive and negative at each temperature studied.
\end{abstract}

\section{INTRODUCTION}

The importance of thermodynamic properties of liquid mixtures in design calculations involving chemical separations, heat transfer, mass transfer and fluid flow have recognized the chemical industries [1]. These properties provide information about the state of components as well as nature and extent of interactions operating among the constituent of mixtures.

To increase the efficiency, quinoline is used in solar cells and also used in dyes. Toluene is a common solvent, able to dissolve paints, paint thinners, silicon sealants, many chemical reactants, rubber, printing ink, adhesives, lacquers, leather tanners and disinfectants. It is used as a solvent for carbon nanomaterials, including nanotubes and fullerenes. Toluene is also used as an octane booster in gasoline fuels used in internal combustion engines. It is recently used as component for jet fuel surrogate blends. Alcohols are self-associated organic liquids and are used as the basic organic compound for the synthesis of other organic compounds. The study of ternary mixtures of quinoline with toluene in methanol would of considerable interest because these mixtures exhibit varying molecular interactions and are important from an industrial point of view.

In continuation of our work on thermodynamic properties of binary and ternary mixtures containing quinolone as one of the component [2,3], we report here ultrasonic velocities and densities of the ternary mixtures of quinoline with toluene in methanol at varying temperatures of $(303.15,308.15,313.15$ and 318.15$) \mathrm{K}$ over the entire range of composition.

Knowledge of various thermodynamic properties of mixtures involving quinoline with selfassociated alcohols and phenols helps in designing an efficient industrial process and also helps in understanding the inter and intra molecular interactions. Several researchers carried out investigations of acoustic and thermodynamical properties of binary mixtures containing phenols [4-7]. As of our knowledge no work is done in ternary mixtures containing quinoline with toluene in methanol.

\section{MATERIALS AND METHODS}

The analytical grade chemicals obtained from SRL Chemicals, Mumbai were used. They were purified by standard procedure [8]. To prepare the mixtures in the required proportions, Job's method of continuous variation was used. The mixtures were preserved in well-stoppard conical 
flasks. After mixing the liquids thoroughly, the flasks were left undisturbed to allow them to attain thermal equilibrium.

Single crystal ultrasonic pulse echo interferometer (Mittal enterprises, India; Model: F-80X) was used for measuring ultrasonic velocities. It consists of a high frequency generator and a measuring cell. The measurements of ultrasonic velocities were made at a fixed frequency of $3 \mathrm{MHz}$. The calibration of the equipment was done by measuring the velocity in benzene and carbon tetrachloride. The results are in good agreement with the literature values [9]. The ultrasonic velocity has an accuracy of $\pm 0.1 \mathrm{~m} \cdot \mathrm{s}^{-1}$. The temperature was controlled by circulating water around the liquid cell from thermostatically controlled constant temperature water bath (accuracy $\pm 0.01 \mathrm{~K}$ ).

\section{THEORY}

The values of Molar volume, $\mathrm{V}_{\mathrm{m}}$ are calculated using standard relation,

$\mathrm{V}_{\mathrm{m}}=\mathrm{M}_{\mathrm{eff}} / \rho$

The acoustic impedance can be calculated by using the relation,

$\mathrm{Z}=\rho \mathrm{u}$

where $M_{\text {eff }}=\left(x_{1} M_{1}+x_{2} M_{2}+x_{3} M_{3}\right), M_{1}, M_{2}$ and $M_{3}$ are the molecular weights of pure components, and $\rho$ is the density of the mixture.

The strength of interaction between the component molecules of ternary mixtures is well reflected in the deviation of the excess functions from ideality [10]. The excess properties such as $V_{m}{ }^{E}, \mathrm{u}^{\mathrm{E}}, \Delta \eta$ and $G^{*}{ }^{E}$ were calculated using the following equations

$V_{m}^{E}=\left(\frac{x_{1} M_{1}+x_{2} M_{2}+x_{3} M_{3}}{\rho}\right)-\left(\frac{x_{1} M_{1}}{\rho_{1}}+\frac{x_{2} M_{2}}{\rho_{2}}+\frac{x_{3} M_{3}}{\rho_{3}}\right)$

$\mathrm{u}^{\mathrm{E}}=\mathrm{u}_{\mathrm{m}}-\left[\mathrm{x}_{1} \mathrm{u}_{1}+\mathrm{x}_{2} \mathrm{u}_{2}+\mathrm{x}_{3} \mathrm{u}_{3}\right]$

$\mathrm{Z}^{\mathrm{E}}=\mathrm{Z}_{\mathrm{m}}-\left[\mathrm{x}_{1} \mathrm{Z}_{1}+\mathrm{x}_{2} \mathrm{Z}_{2}+\mathrm{x}_{3} \mathrm{Z}_{3}\right]$

In equations (3), (4) and (5) $\rho, \mathrm{u}_{\mathrm{m}}, \mathrm{Z}_{\mathrm{m}}$ and $\mathrm{V}_{\mathrm{m}}$ are the density, ultrasonic velocity, acoustic impedance and molar volume of the mixture.

\section{RESULTS AND DISCUSSION}

The experimental values of ultrasonic velocity $(u)$ and density $(\rho)$ for the ternary mixtures at temperatures $\mathrm{T}=(303.15,308.15,313.15$ and 318.15$) \mathrm{K}$ are given as a function of mole fraction in Table 1. From the table it is observed that the ultrasonic velocity and density values increase nonlinearly with the mole fraction of first component. This trend suggests the possibility of intermolecular interactions between the components of the mixtures. The increasing values of density shows that the addition of first component makes the system to be more compact, thereby showing the attractive type interactions between the components. As the medium becomes more and more compact, velocity also increases as is observed in this case. 
Table 1: Ultrasonic velocities $(u)$ and densities $(\rho)$ for the ternary mixtures at $\mathrm{T}=(303.15,308.15$, 313.15 , and 318.15$) \mathrm{K}$

\begin{tabular}{|c|c|c|c|c|c|c|c|}
\hline $\mathbf{x}_{1}$ & $\mathbf{x}_{\mathbf{3}}$ & $\begin{array}{c}\mathbf{u} \\
\left(\mathrm{m} \mathrm{s}^{-1}\right)\end{array}$ & $\begin{array}{c}\rho \\
\left(\mathrm{Kg} \mathrm{m}^{-3}\right)\end{array}$ & $\mathbf{x}_{1}$ & $\mathbf{x}_{\mathbf{3}}$ & $\begin{array}{c}\mathbf{u} \\
\left(\mathrm{m} \mathrm{s}^{-1}\right)\end{array}$ & $\begin{array}{c}\rho \\
\left(\mathrm{Kg} \mathrm{m}^{-3}\right)\end{array}$ \\
\hline \multicolumn{8}{|c|}{$\mathrm{T}=303.15 \mathrm{~K}$} \\
\hline 0.0000 & 0.7713 & 1193.68 & 825.63 & 0.4039 & 0.3576 & 1333.15 & 1002.86 \\
\hline 0.0781 & 0.6913 & 1215.15 & 870.41 & 0.4889 & 0.2705 & 1375.94 & 1025.16 \\
\hline 0.1575 & 0.6100 & 1243.68 & 915.40 & 0.5754 & 0.1819 & 1416.31 & 1044.57 \\
\hline 0.2382 & 0.5273 & 1268.1 & 946.62 & 0.6634 & 0.0918 & 1470.78 & 1065.00 \\
\hline 0.3204 & 0.4431 & 1299.73 & 978.60 & 0.7530 & 0.0000 & 1527.10 & 1082.54 \\
\hline \multicolumn{8}{|c|}{$\mathrm{T}=308.15 \mathrm{~K}$} \\
\hline 0.0000 & 0.7713 & 1130.52 & 807.40 & 0.4039 & 0.3576 & 1282.94 & 986.71 \\
\hline 0.0781 & 0.6913 & 1156.84 & 852.73 & 0.4889 & 0.2705 & 1323.63 & 1009.76 \\
\hline 0.1575 & 0.6100 & 1183.68 & 897.87 & 0.5754 & 0.1819 & 1374.63 & 1030.25 \\
\hline 0.2382 & 0.5273 & 1214.73 & 929.76 & 0.6634 & 0.0918 & 1432.47 & 1051.62 \\
\hline 0.3204 & 0.4431 & 1246.84 & 959.58 & 0.7530 & 0.0000 & 1520.94 & 1072.32 \\
\hline \multicolumn{8}{|c|}{$\mathrm{T}=313.15 \mathrm{~K}$} \\
\hline 0.0000 & 0.7713 & 1100.78 & 797.56 & 0.4039 & 0.3576 & 1264.21 & 970.42 \\
\hline 0.0781 & 0.6913 & 1135.15 & 838.58 & 0.4889 & 0.2705 & 1302.63 & 992.56 \\
\hline 0.1575 & 0.6100 & 1164.31 & 880.22 & 0.5754 & 0.1819 & 1355.84 & 1016.92 \\
\hline 0.2382 & 0.5273 & 1195.63 & 913.49 & 0.6634 & 0.0918 & 1412.63 & 1040.19 \\
\hline 0.3204 & 0.4431 & 1226.31 & 944.49 & 0.7530 & 0.0000 & 1511.47 & 1066.08 \\
\hline \multicolumn{8}{|c|}{$\mathrm{T}=318.15 \mathrm{~K}$} \\
\hline 0.0000 & 0.7713 & 1078.94 & 784.04 & 0.4039 & 0.3576 & 1242 & 949.61 \\
\hline 0.0781 & 0.6913 & 1110.52 & 819.65 & 0.4889 & 0.2705 & 1285 & 976.34 \\
\hline 0.1575 & 0.6100 & 1145.36 & 860.76 & 0.5754 & 0.1819 & 1332.78 & 1001.09 \\
\hline 0.2382 & 0.5273 & 1176.31 & 893.03 & 0.6634 & 0.0918 & 1395.36 & 1030.83 \\
\hline 0.3204 & 0.4431 & 1210.15 & 924.22 & 0.7530 & 0.0000 & 1505.10 & 1061.86 \\
\hline
\end{tabular}

The variations of $\mathrm{V}_{\mathrm{m}}^{\mathrm{E}}, \mathrm{u}^{\mathrm{E}}$ and $\mathrm{Z}^{\mathrm{E}}$ with the mole fraction of quinoline for toluene in methanol at $(303.15,308.15,313.15,318.15) \mathrm{K}$ are plotted in Fig. 1, Fig. 2 and Fig. 3 respectively. 


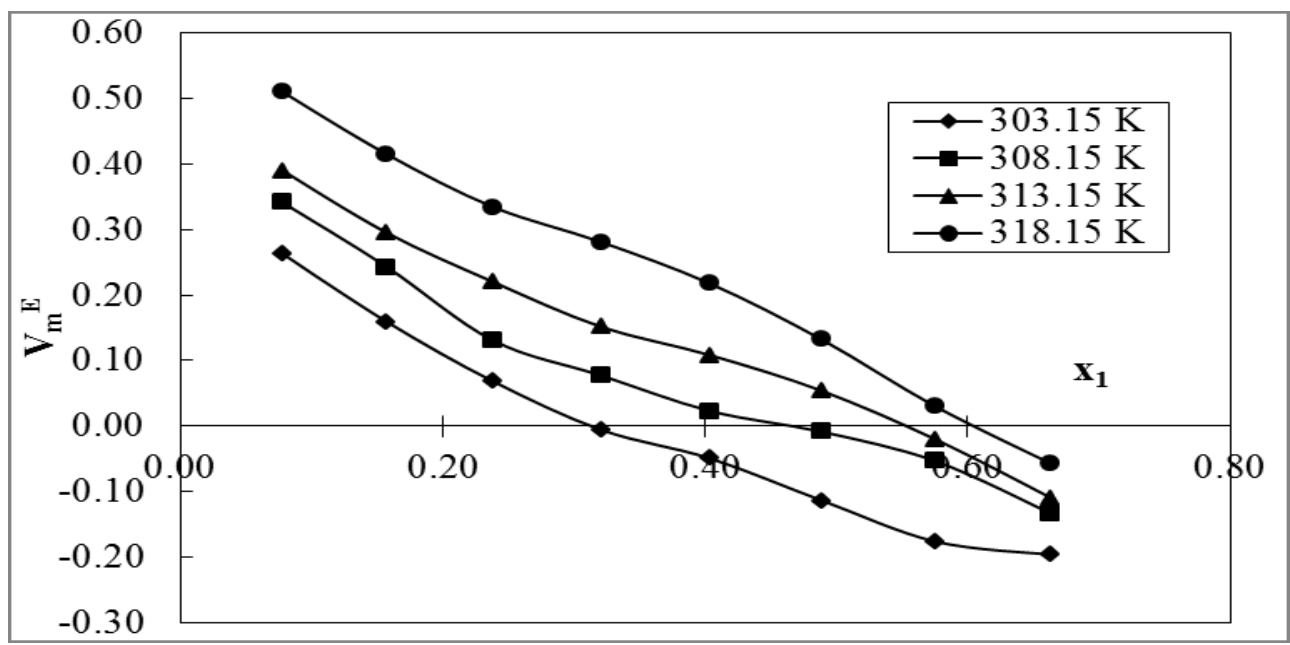

Figure 1: Variation of excess molar volume, $\mathrm{V}_{\mathrm{m}}^{\mathrm{E}}$, with mole fraction, $\mathrm{x}_{1}$, for Quinoline+ methanol+toluene mixtures at different temperatures

Excess volume data are useful in the conversion of excess thermodynamic functions determined at constant pressure to the condition of mixing at constant volume, in determining composition from density measurements on mixtures, and in industrial technology. Thus measurements of excess volumes continue to be an area of active interest and this is reflected by the enormous amount of data published over the last decade.

The results shown in Fig. 1 indicate that $\mathrm{V}_{\mathrm{m}}{ }^{\mathrm{E}}$ values are positive at lower mole fractions and negative at higher mole fractions at all temperatures reported. Generally, dispersive forces and improper interstitial accommodation of molecules of a ternary mixture will be reflected in positive $\mathrm{V}_{\mathrm{m}}{ }^{\mathrm{E}}[11,12]$. However, strong interactions taking place between unlike molecules through charge transfer forces, the formation of new hydrogen bonds and proper interstitial accommodation will yield negative $\mathrm{V}_{\mathrm{m}}^{\mathrm{E}}$ [13]. The dispersive forces and improper interstitial accommodation of molecules of a ternary mixture will be reflected in positive $\mathrm{V}_{\mathrm{m}}^{\mathrm{E}}$.

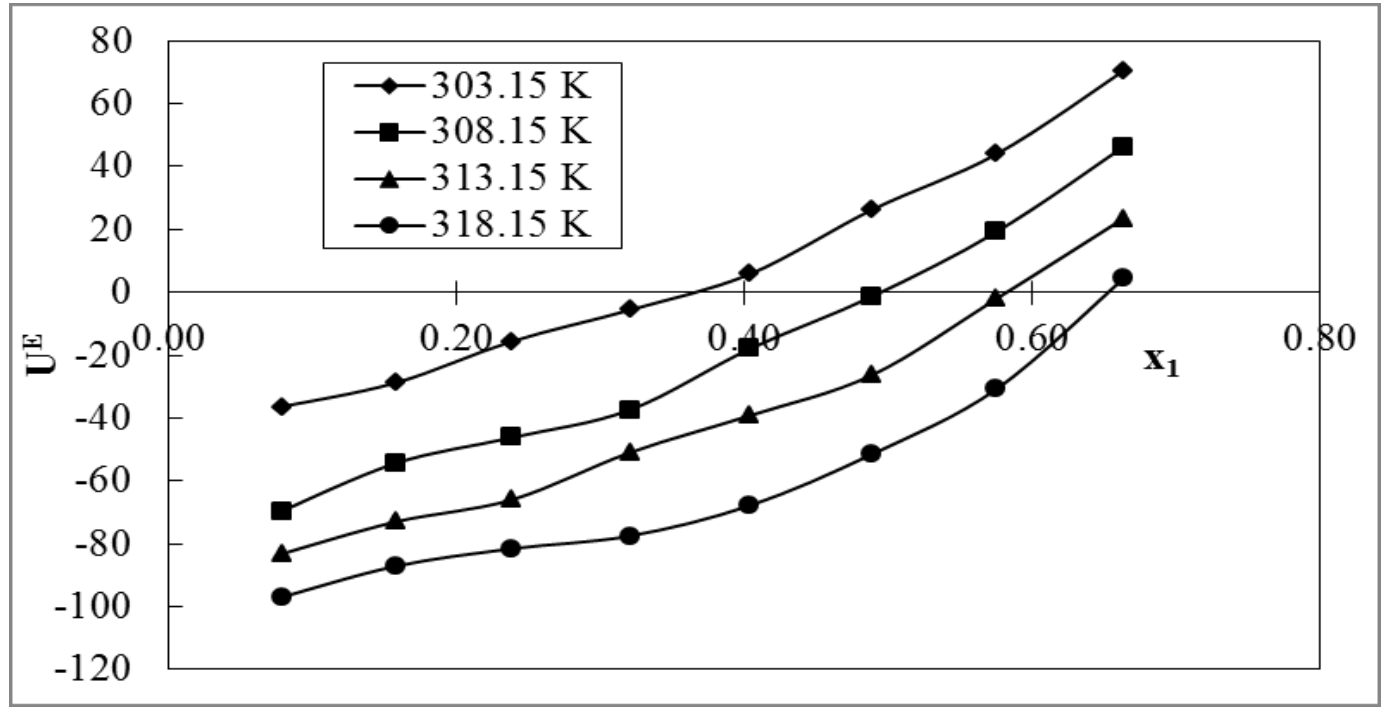

Figure 2: Variation of excess ultrasonic velocity, $\mathrm{u}^{\mathrm{E}}$, with mole fraction, $\mathrm{x}_{1}$, for Quinoline + methanol + toluene mixtures at different temperatures

The intermolecular interaction occurring in the liquid mixtures might result in the decrease of the interspace between molecules and this might lead to a decrease in intermolecular free length producing negative values of the excess intermolecular free length. The decrease of intermolecular free length results in a decrease of compressibility and an increase of velocity. 
From Fig. 2 it is observed that as the mole fraction of quinoline increases, the $\mathrm{u}^{\mathrm{E}}$ values becomes positive and at all temperatures. The liquid mixture volume depends upon intermolecular interactions as well as structural arrangement [14]. An increase in the strength of the heteromolecular forces manifests in a decrease in adiabatic compressibility and volume and, hence the size of cluster. Thus, negative $\mathrm{V}_{\mathrm{m}}{ }^{\mathrm{E}}$ values account for the strong interactions between the unlike molecules. Thus excess molar volume and excess ultrasonic velocity complements the presence of strong interactions in the system and at lower mole fraction of quinoline.

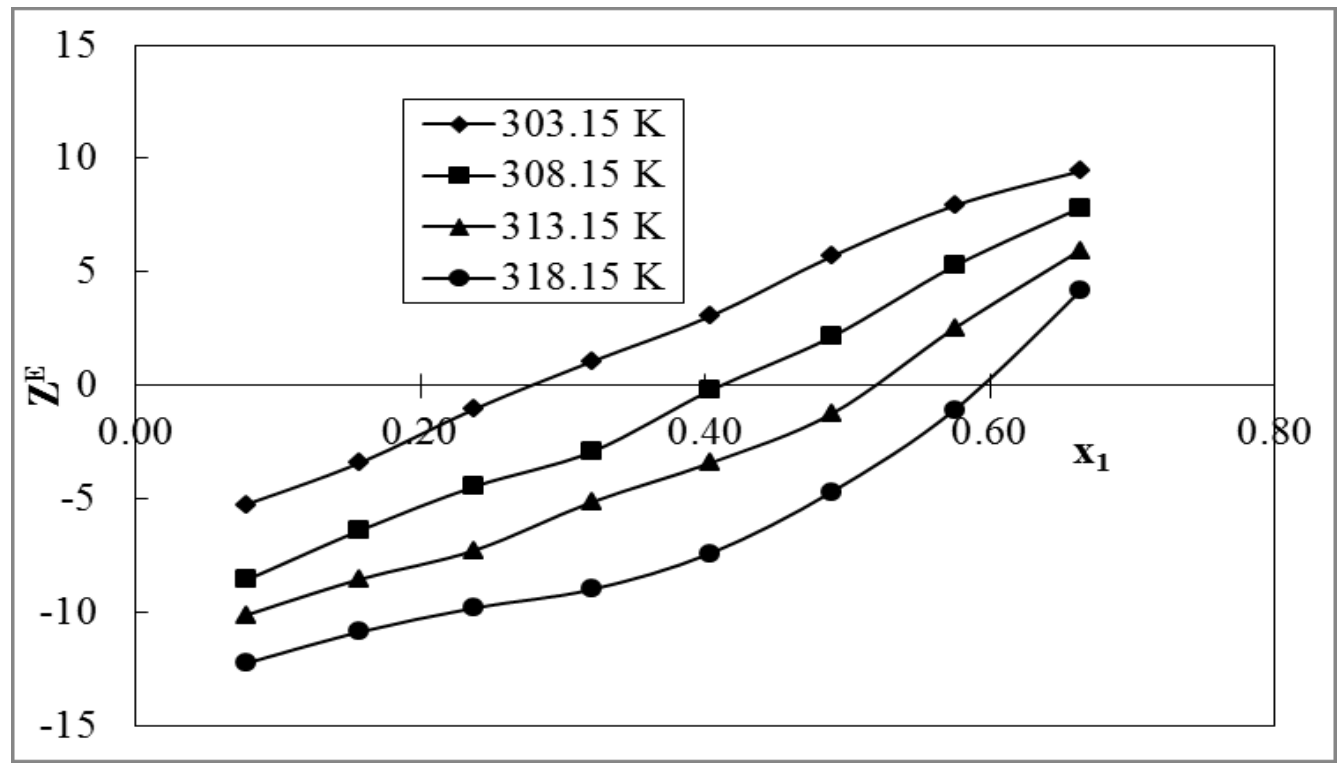

Figure 3: Variation of excess acoustic impedance, $Z^{\mathrm{E}}$, with mole fraction, $\mathrm{x}_{1}$, for Quinoline + methanol + toluene mixtures at different temperatures

From Fig. 3 it is observed that the excess acoustic impedance values for all the mixtures are positive at higher mole fraction of quinoline and negative at lower mole fractions. The positive deviations in $Z^{\mathrm{E}}$ from linear dependence suggest the presence of strong interactions between the component molecules [15]. It is also observed from Fig. 3 that as the temperature increases the $Z^{\mathrm{E}}$ values become less positive and it shows that the interactions become weaker with increase of temperature.

From all the above results we may conclude that there exist stong interactions between the molecules of the mixtures in the system choosen for the study.

\section{Conclusion}

Ultrasonic velocity and density values are measured in pure components and ternary mixtures of quinoline + methanol + toluene at temperatures $303.15,308.15,313.15$ and $318.15 \mathrm{~K}$ over the entire composition range. The observed positive and negative values of $V_{m}{ }^{E}, u^{E}$ and $Z^{E}$ suggest the presence of molecular interactions in the present system.

\section{References}

[1] M.B. Ewing, B.J. Levian, K.N. Marsh, J. Chem. Thermodyn. 2(5) (1970) 689-695.

[2] K. Narendra, Ch. Srinivsu, Ch. Kalpana, P. Narayanamurthy, J. Therm. Anal. Calorim. 107 (2012) 25-30.

[3] Sk. Fakruddin, K. Narendra and Ch. Srinivasu, Journal of Chemical and Pharmaceutical Research 4(7) (2012) 3606-3609.

[4] G. Mahendran, and L. Palaniappan, 2011. Indian J Pure Appl Phys. 49 (2011) 803-808. 
[5] Ch. Kavitha, A. Ratnakar, M. Sri Lakshmi, K. Narendra, Journal of Applicable Chemistry. 3(1) (2014) 360-365.

[6] D. Archana, and M. Singh, Indian J Chem. 46A (2007) 789-794.

[7] A. Ali, A. Yasmin, A.K. Nain, Indian J. Pure Appl. Phys. 40 (2002) 315-322.

[8] D.D. Perrin, W.L.F. Armarego, Purification of Lab. Chem., third ed., Pergamon Press, Oxford, 1980.

[9] D.R. Lide, CRC Handbook of Chemistry and Physics, $76^{\text {th }}$ ed., CRC Press, 1995.

[10] J.D. Pandey, R.D. Rai, R.K. Shukla, A.K. Shukla, N. Mishra, Indian J. Pure Appl. Phys. 31 (1993) 84.

[11] G.V. Rama Rao, A. Viswanath Sarma, C. Rambabu, Indian J. Chem. 43A (2004) 25182528

[12] T. Savitha Jyostna, Satyanarayana, Indian J. Chem. 44A (2005) 1365-1371.

[13] S. Chauhan, S. Syal, M.S. Chauhan, Indian J Pure Appl Phys. 32 (1994) 186.

[14] T. Sumathi, J. Uma Maheswari, Indian J. Pure Appl. Phys. 47 (2009) 782-786.

[15] K.V. Ramana Reddy, K.S. Reddy, A. Krishnaiah, J. Chem. Eng. Data. 39 (1994) 615-617. 